\title{
END EFFECTS IN \\ A TWO DIMENSIONAL POTENTIAL PROBLEM FOR CLOSEIYY SPACED RECTANGULAR PLATES
}

\author{
N. G. BARTON 1
}

(Received 13 December 1983; revised 27 February 1984)

\begin{abstract}
This paper presents a conformal mapping solution of Laplace's equation in the two dimensional region exterior to two rectangular plates or electrodes at different potentials. Plates with finite and semi-infinite lengths are considered separately and particular emphasis is placed upon the case when the separation between the plates is small. The key results of the paper are expressions for the integral of the square of the normal field along the mid-line between the plates. This integral is of importance in certain gaseous conductor experiments that are sufficiently accurate for a consideration of end effects to be necessary. For small gaps, the dominant end correction to the integral is linear with the gap width. It is also shown that, for small gaps, the simplified (semi-infinite) geometry gives essentially the same value for the integral as the full (finite plate length) geometry.
\end{abstract}

\section{Introduction}

This paper investigates the two dimensional solution of Laplace's equation between two equal and closely spaced rectangular plates (or electrodes or pole pieces). The problem has a number of possible applications in the physical and engineering sciences, and it was brought to the author's attention by a physical chemist who determined properties of gases by shining a laser down the mid-line between two closely spaced parallel electrodes maintained at different potentials. This experiment related certain gas properties to the integral along the mid-line of the square of the normal electric field, and it was sufficiently accurate that a consideration of end effects was warranted even though the quotient (gap

\footnotetext{
${ }^{1}$ C.S.I.R.O. Division of Mathematics and Statistics, P. O. Box 218, Lindfield, N.S.W., 2070.

(C) Copyright Australian Mathematical Society 1985, Serial-fee code 0334-2700/85
} 
width/plate length) was about $1 / 100$. If end effects were neglected, the integral would, of course, take the value (potential difference between plates/plate spacing) ${ }^{2} \times$ length of plate. The concept of end corrections is an old one in other branches of physics, and perhaps the best known example is Rayleigh's open end correction for sound waves in pipes [8].

The problem of calculating the potential between two equal semi-infinite rectangular pole pieces can be solved using a Schwarz-Christoffel conformal map, described in reference [7]. The differential equation for the transformation was originally described by Riemann and Weber [9], and Davy [4] has given many more details of the solution whilst not presenting the analysis required for the present (small gap) application.

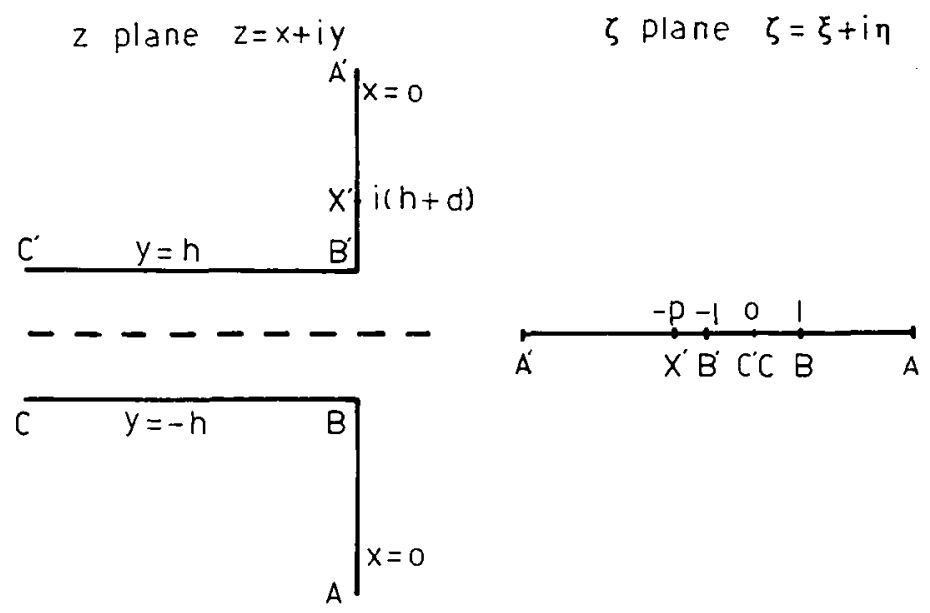

(a)

(b)

FIGURE 1. Illustrating the geometry for the problem discussed in Sections 2 and 3. The point $X^{\prime}$ at $z=i(h+d)$ is concerned with the boundary conditions discussed in Section 3.3.

A rather simpler problem is investigated first of all, however, before adapting the Riemann-Weber-Davy transformation to the small gap approximation. In this simplified problem, the electrodes are allowed to become of semi-infinite extent in two directions as shown in Figure 1(a). The conformal map which transforms the domain to the upper half of the $\zeta$ plane is described in Section 2 , and the potential is calculated for various boundary conditions in Section 3. Then the integral mentioned above is evaluated using the small gap approximation, and the correction terms for end effects are presented. Apart from exponentially small terms, the correction depends linearly on the gap width.

In Section 4, the Riemann-Weber-Davy transformation is summarised and, in Section 5, the small gap approximation to the integral in question is derived for one simple boundary condition. Apart from insignificant exponentially small 
terms, the small gap result is identical to that obtained in Section 3 using the simpler geometry. It is suggested, therefore, that the simpler problem furnishes an excellent approximation to the full problem, and that investigations of the effects of changed boundary conditions or perturbations to the geometry should be carried out using the simpler geometry. This would appear to be a most useful conclusion for any subsequent work.

\section{Conformal mapping for the simplified problem}

Suppose that the variables $x$ and $y$ are rendered dimensionless using the length of the plate; the dimensionless gap width is $2 h$ as shown in Figure 1(a). This Section contains details of the transformation which maps the simplified domain shown in Figure 1(a) onto the upper half plane shown in Figure 1(b). The differential equation defining the Schwarz-Christoffel transformation is (see, for example, Carrier, et al. [2])

$$
\frac{d z}{d \zeta}=\frac{K\left(\zeta^{2}-1\right)^{1 / 2}}{\zeta}
$$

which may be integrated to give

$$
z=-i K\left\{\left(1-\zeta^{2}\right)^{1 / 2}-\tanh ^{-1}\left(1-\zeta^{2}\right)^{1 / 2}\right\}+C .
$$

The constants $K$ and $C$ are determined by ensuring that $z=i$ maps to $\zeta=-1$ and $z=-i h$ maps to $\zeta=1$. The branch of $\tanh ^{-1}\left(1-\zeta^{2}\right)^{1 / 2}$ is determined by using $-\tanh ^{-1}\left(1-\zeta^{2}\right)^{1 / 2}=\ln \zeta-\ln \left[1+\left(1-\zeta^{2}\right)^{1 / 2}\right]$ and the principal branch for $\ln \zeta$; substitution of $z= \pm$ ih then gives

$$
i h=-i K\{0+\ln (-1)-\ln 1\}+C, \quad-i h=-i K\{0+\ln 1-\ln 1\}+C,
$$

from which it follows that the constants $C$ and $K$ are

$$
C=-i h, \quad K=2 i h / \pi \text {. }
$$

Thus the conformal map is given by either of the equivalent forms

$$
\begin{gathered}
z=\frac{2 h}{\pi}\left\{-\frac{i \pi}{2}+\left(1-\zeta^{2}\right)^{1 / 2}-\tanh ^{-1}\left(1-\zeta^{2}\right)^{1 / 2}\right\} \\
z=\frac{2 h}{\pi}\left\{-\frac{i \pi}{2}+\left(1-\zeta^{2}\right)^{1 / 2}+\ln \zeta-\ln \left[1+\left(1-\zeta^{2}\right)^{1 / 2}\right]\right\} .
\end{gathered}
$$

The key results of this work concern the integral along the mid-line between the plates of the square of the normal field, that is,

$$
I=\int_{-\infty}^{\infty}\left(\frac{\partial \phi}{\partial y}\right)_{y=0}^{2} d x
$$


This form of the integral is not appropriate for the semi-infinite geometry under consideration; rather it should be evaluated as

$$
I=2 \int_{-1 / 2}^{\infty}\left(\frac{\partial \phi}{\partial y}\right)_{y=0}^{2} d x
$$

where the co-ordinates $x$ and $y$ are as shown in Figure 1(a) and the plate has length 1 by the assumed nondimensionalization. This integral $(2.3 \mathrm{~b})$ is most conveniently evaluated in the following way. Suppose that $G(z)$ is an analytic function with $\phi$ as its imaginary part. Then we have

$$
\frac{\partial \phi}{\partial y}=\operatorname{Im}\left(\frac{\partial G}{\partial y}\right)=\operatorname{Im}\left(i \frac{d G}{d z}\right)=\operatorname{Re}\left(\frac{d G}{d z}\right)=\operatorname{Re}\left(\frac{d G}{d \zeta} \frac{d \zeta}{d z}\right)
$$

and the integral $(2.3 \mathrm{~b})$ becomes

$$
I=2 \int_{\zeta(-1 / 2)}^{\zeta(\infty)}\left\{\operatorname{Re}\left(\frac{d G}{d \zeta} \frac{d \zeta}{d z}\right)\right\}^{2} \frac{d z}{d \zeta} d \zeta
$$

Now the line $y=0$ in the $z$ plane maps onto the line $\xi=\operatorname{Re}(\zeta)=0$ in the $\zeta$ plane, and this is the natural choice for the path of integration in integral (2.4). On this line, the integral $(2.3 \mathrm{~b})$ becomes

$$
I=-2 i \int_{\eta(-1 / 2)}^{\eta(\infty)}\left\{\operatorname{Im} \frac{d G}{d \zeta}\right\}^{2} \frac{d \zeta}{d z} d \eta
$$

where $-i d \zeta / d z$ is real, positive and of course independent of the boundary conditions applied on $\phi$.

To complete this section, the terminals $\eta\left(-\frac{1}{2}\right)$ and $\eta(\infty)$ in the integral (2.5) are calculated for later use. Using expression (2.2b), $\eta\left(-\frac{1}{2}\right)$ is the root of

$$
-\frac{1}{2}=\frac{2 h}{\pi}\left\{-\frac{i \pi}{2}+\left(1+\eta^{2}\right)^{1 / 2}+\ln (i \eta)-\ln \left[1+\left(1+\eta^{2}\right)^{1 / 2}\right]\right\}
$$

and it is, approximately,

$$
\eta\left(-\frac{1}{2}\right)=2 \exp \left\{-\left(\frac{\pi}{4 h}+1\right)-\exp \left\{-2\left(\frac{\pi}{4 h}+1\right)\right\}\right\}
$$

for $h$ small, the upper terminal $\eta(\infty)$ is easily seen to be

$$
\eta(\infty)=\infty
$$

\section{Results for the simplified problem}

The integral (2.3b) is evaluated in this section when the harmonic function $\phi$ satisfies boundary conditions of the form

$$
\phi(\xi, 0)= \begin{cases}0, & -\infty<\xi<-\xi_{1}, \\ v, & -\xi_{1}<\xi<-\xi_{2}, \\ 0, & -\xi_{2}<\xi<\infty\end{cases}
$$


Some thought shows that the analytic function $G(\zeta)$ whose imaginary part $\phi$ satisfies the above conditions is

$$
G=\frac{V}{\pi} \log \left(\frac{\zeta+\xi_{2}}{\zeta+\xi_{1}}\right)
$$

so that

$$
\operatorname{Im}\left(\frac{d G}{d \zeta}\right)=\frac{V}{\pi}\left(\frac{\pi}{\eta^{2}+\xi_{1}^{2}}-\frac{\pi}{\eta^{2}+\xi_{2}^{2}}\right)
$$

on the axis $\zeta=0$. Then, bearing in mind that $d z / d \zeta$ is defined by (2.1) with $K=2 i h / \pi$, the integral (2.5) for $I$ becomes

$$
I=\frac{V^{2}}{\pi h} \int_{\eta(-1 / 2)}^{\eta(\infty)}\left(\frac{\eta}{\eta^{2}+\xi_{1}^{2}}+\frac{\eta}{\eta^{2}+\xi_{2}^{2}}\right)^{2} \frac{\eta}{\left(1+\eta^{2}\right)^{1 / 2}} d \eta .
$$

3.1. First suppose that the entire upper plate in Figure 1(a) is maintained at a potential $V$ with respect to the lower plate. Then $\xi_{1}$ and $\xi_{2}$ are set to be $\xi_{1}=\infty$, $\xi_{2}=0$, and (3.3) reduces to

$$
I=\frac{V^{2}}{\pi h} \int_{\eta(-1 / 2)}^{\eta(\infty)} \frac{d \eta}{\eta\left(1+\eta^{2}\right)^{1 / 2}}
$$

or

$$
I=\frac{V^{2}}{\pi h}\left[\ln \eta-\ln \left(1+\left(1+\eta^{2}\right)^{1 / 2}\right)\right]_{\eta(-1 / 2)}^{\eta(\infty)}
$$

from Gradshteyn and Rhyzhik [6, equation 2.266]. Straightforward manipulations using the expressions $(2.6 \mathrm{a}, \mathrm{b})$ then establish that, for $h$ small,

$$
I=\frac{V^{2}}{4 h^{2}}\left[1+\frac{4 h}{\pi}+\frac{8 h}{\pi} \exp \left\{-2\left(\frac{\pi}{4 h}+1\right)\right\}+\cdots\right] \text {. }
$$

3.2. Now suppose that only the boundary segment $C^{\prime} B^{\prime}$ of the upper plate has potential $V$ so that $\xi_{1}=1$ and $\xi_{2}=0$. The integral (3.3) now becomes

$$
I=\frac{V^{2}}{\pi h} \int_{\eta(-1 / 2)}^{\eta(\infty)} \frac{d \eta}{\eta\left(1+\eta^{2}\right)^{5 / 2}}
$$

or

$$
I=\frac{V^{2}}{\pi h}\left[\ln \eta-\ln \left(1+\left(1+\eta^{2}\right)^{1 / 2}\right)+\left(1+\eta^{2}\right)^{-1 / 2}+\frac{1}{3}\left(1+\eta^{2}\right)^{-3 / 2}\right]_{\eta(-1 / 2)}^{\eta(\infty)}
$$

by [6, equation $2.248(2)]$. For $h$ small, the results $(2.6 \mathrm{a}, \mathrm{b})$ now give

$$
I=\frac{V^{2}}{4 h^{2}}\left[1-\frac{4 h}{3 \pi}+\frac{24 h}{\pi} \exp \left\{-2\left(\frac{\pi}{4 h}+1\right)\right\}+\cdots\right] .
$$


3.3. Finally, suppose that the potential $V$ is applied at the boundary segment $C^{\prime} B^{\prime} X^{\prime}$ in Figure 1(a). Then $\xi_{1}$ and $\xi_{2}$ are set to be $\xi_{1}=p, \xi_{2}=0$ and the integral (3.3) reduces to

$$
I=\frac{V^{2} p^{4}}{\pi h} \int_{\eta(-1 / 2)}^{\eta(\infty)} \frac{d \eta}{\eta\left(\eta^{2}+p^{2}\right)\left(1+\eta^{2}\right)^{1 / 2}} .
$$

Here, the constant $p$ depends on the length $d$ (see Figure 1(a)) and is given by the root of the equation

$$
\left(p^{2}-1\right)^{1 / 2}-\tan ^{-1}\left(p^{2}-1\right)^{1 / 2}+\pi=\pi(1+d / 2 h)
$$

obtained by substituting $z=i(h+d), \zeta=-p$ in expression $(2.2 \mathrm{~b})$. In general, the roots of equation (3.7) would have to be found numerically, although the approximation

$$
p=1+\frac{1}{2}\left[\frac{3 \pi d}{2 h}\right]^{2 / 3}+\cdots
$$

can be used when $d / 2 h$ is small.

The integral (3.6) can be evaluated by substituting $\eta=1 / \sinh v$ and using results $(2.458,2.459)$ in reference [6]; it is found to be

$$
\begin{aligned}
I=\frac{V^{2}}{\pi h}\left[\ln \eta-\ln \left(1+\left(1+\eta^{2}\right)^{1 / 2}\right)+\frac{3 p^{2}-2}{2\left(p^{2}-1\right)^{3 / 2}}\right. & \tan ^{-1} \frac{\left(p^{2}-1\right)^{1 / 2}}{\left(1+\eta^{2}\right)^{1 / 2}} \\
& \left.-\frac{p^{2}\left(1+\eta^{2}\right)^{1 / 2}}{2\left(p^{2}-1\right)\left(\eta^{2}+p^{2}\right)}\right]_{\eta(-1 / 2)}^{\eta(\infty)}
\end{aligned}
$$

Much would remain to be done if this result were to interpreted for general $h$ and $p$; however, the approximation

$$
\begin{aligned}
I=\frac{V^{2}}{4 h^{2}}(1 & -\frac{4 h}{\pi}\left[\frac{1}{3}-\frac{2}{5}\left(p^{2}-1\right)\right] \\
& \left.+\frac{4 h}{\pi}\left[6-4\left(p^{2}-1\right)\right] \exp \left\{-2\left(\frac{\pi}{4 h}+1\right)\right\}+\cdots\right)
\end{aligned}
$$

can be deduced for $h$ and $\left(p^{2}-1\right)$ small.

The approximations $(3.3,3.5,3.8)$ provide the key results of this section. They show that the contributions to the integral $I$ by end effects are of $O(h)$ times the dominant term $I=V^{2} /(2 h)^{2}$. The constant in this $O(h)$ correction term is $4 / \pi$ for the boundary conditions of Section 3.1 and $-4 / 3 \pi$ for those of Section 3.2: evidently, a reduction in end effects can be achieved merely by changing the boundary conditions. 


\section{Conformal mapping for the unsimplified problem}

In this section, the Riemann-Weber-Davy transformation which maps the domain shown in Figure 2(a) onto the upper half plane is summarized. The presentation follows that of Davy [4] except that the points $z=-b \pm i \infty$ are now mapped to $\zeta=0$, whereas Davy mapped $z=b \pm i \infty$ to $\zeta=0$.

$z$ plane $z=x+i y$

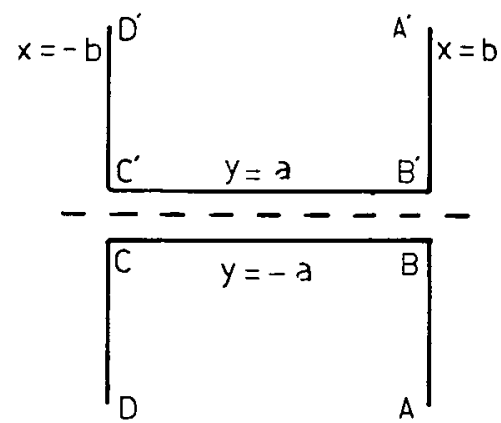

(a) $\zeta$ plane $\zeta=\xi+i \eta$

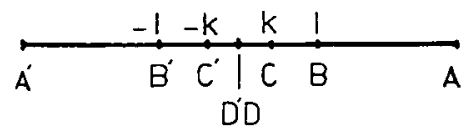

(b)

FIGURE 2. Illustrating the geometry for the finite plate length geometry studied in Sections 4 and 5.

The differential equation describing the tranformation is

$$
\frac{d z}{d \zeta}=\frac{S\left(\zeta^{2}-k^{2}\right)^{1 / 2}\left(\zeta^{2}-1\right)^{1 / 2}}{\zeta^{2}}
$$

where the points $z=b \pm i a$ map to $\zeta=\mp 1$ and $z=-b \pm i a$ map to $\zeta=\mp k$ $(0<k<1)$. The differential equation is integrated by substituting $\zeta=1 / \mathrm{sn} u$ and making $k$ the modulus of the elliptic functions used. It is found that the transformation becomes

$$
\frac{d z}{d u}=-S\left\{\frac{1}{\operatorname{sn}^{2} u}-\left(1+k^{2}\right)+k^{2} \operatorname{sn}^{2} u\right\}
$$

and this may be integrated to give

$$
z=-S\left\{u\left(k^{\prime 2}-\frac{2 E}{K}\right)-2 Z(u)-\frac{\operatorname{cn} u \operatorname{dn} u}{\operatorname{sn} u}\right\}+T
$$

where $k^{\prime 2}=1-k^{2}, K$ and $E$ are the complete elliptic integrals of the first and second kinds, $Z(u)$ is the Jacobian elliptic function and $T$ is a constant of integration. The constants $S, T$ and $k$ are determined by substituting the known 
values for the points $B^{\prime}$ and $C^{\prime}$. Using the values described in Table 1, this procedure gives

$$
\begin{gathered}
b+i a=-S\left\{-K k^{\prime 2}+2 E\right\}+T \\
-b+i a=-S\left\{-\left(K+i K^{\prime}\right) k^{\prime 2}+2\left[E+i\left(K^{\prime}-E^{\prime}\right)\right]\right\}+T
\end{gathered}
$$

where $K^{\prime}$ and $E^{\prime}$ are the same functions of $k^{\prime}$ as $K$ and $E$ are of $k$. An examination of the real and imaginary parts of these expressions gives the relationships

$$
\begin{aligned}
& -S=\frac{a i}{2 E-k^{\prime 2} K}, T=-b, \\
& b=\frac{a\left(K^{\prime} k^{\prime 2}-2 K^{\prime}+2 E^{\prime}\right)}{2\left(K k^{\prime 2}-2 E\right)}
\end{aligned}
$$

previously obtained by Davy [4].

TABLE 1. Certain values associated with the tranformation.

\begin{tabular}{|c|c|c|c|c|c|}
\hline Point & $z$ & $\zeta$ & sn $u$ & $u$ & $E(u)$ \\
\hline$B^{\prime}$ & $b+i a$ & -1 & -1 & $-K$ & $-E$ \\
\hline$C^{\prime}$ & $-b+i a$ & $-k$ & $-1 / k$ & $-\left(K+i K^{\prime}\right)$ & $-E-i\left(K^{\prime}-E^{\prime}\right)$ \\
\hline
\end{tabular}

It is noteworthy that Davy [4] has described further properties of the potential solution-in particular, he has plotted equipotentials and calculated the electric field for special values of $a$ and $b$, and he has made experiments which confirm the theoretical results. The small gap approximation was not included in his work, however.

\section{Results for the unsimplified problem}

The integral to be evaluated in this section is (2.3a) which is seen to be equivalent to

$$
I=-i \int_{0}^{\infty}\left\{\operatorname{Im} \frac{d G}{d \zeta}\right\}^{2} \frac{d \zeta}{d z} d \eta
$$

by using the same arguments which led to equation (2.5). If the entire upper plate in Figure 2(a) is considered to be at potential $V$ with respect to the lower plate, then the constants $\xi_{1}$ and $\xi_{2}$ in the definition (3.2) for $G(\zeta)$ are set to be $\xi_{1}=\infty$, $\xi_{2}=0$. In this case, the integral above reduces to

$$
I=\frac{i V^{2}}{\pi^{2} s} \int_{0}^{\infty} \frac{d \eta}{\left(k^{2}+\eta^{2}\right)^{1 / 2}\left(1+\eta^{2}\right)^{1 / 2}}
$$


which, by reference [6, equation $3.152(1)]$, is seen to be

$$
I=\frac{i V^{2} K^{\prime}}{\pi^{2} S}
$$

It remains to calculate the small gap approximation to this result. If the values $b=\frac{1}{2}, a=h$ are chosen in order to correspond with the nondimensionalization used in Sections 2 and 3, expression (4.4b) requires that

$$
\frac{1}{2 h}=\frac{K^{\prime} k^{\prime 2}-2 K^{\prime}+2 E^{\prime}}{2\left(K k^{\prime 2}-2 E\right)}
$$

which may be re-arranged to give

$$
k^{\prime 2}=\frac{2\left[E+h\left(E^{\prime}-K^{\prime}\right)\right]}{K-h K^{\prime}} .
$$

Some thought shows that $k \rightarrow 0$ and $h \rightarrow 0$, and so the root of equation (5.2) may be obtained using expansions for small $k$. As it turns out, the root for $k$ is very small indeed, thus ensuring that the procedure is consistent.

The expansions are carried out using $m=k^{2}$. Abramowitz and Stegun [1, equations 17.3.11, 17.3.12] give that

$$
\begin{aligned}
& K=\frac{\pi}{2}\left[1+\frac{m}{4}+\frac{9 m^{2}}{64}+\cdots\right], \\
& E=\frac{\pi}{2}\left[1-\frac{m}{4}-\frac{3 m^{2}}{64}+\cdots\right]
\end{aligned}
$$

also, the nome $q[1$, equations $17.3 .17,17.3 .21]$

$$
q=\frac{m}{16}+\frac{m^{2}}{32}+\frac{21 m^{3}}{1024}+\cdots
$$

is used in the definition of $K^{\prime}$,

$$
K^{\prime}=\frac{-K}{\pi} \ln q
$$

Finally, $E^{\prime}$ is obtained using Legendre's identity [1, equation 17.3.13]

$$
E^{\prime}=\frac{\pi}{2 K}+\frac{K^{\prime}}{K}(K-E) \text {. }
$$

The laborious substitution of these expressions for $K, E, K^{\prime}$ and $E^{\prime}$ in equation (5.2) finally reveals that the root for $k$ is $k=\sqrt{m}$ where, to an excellent approximation.

$$
m=16 \exp \left\{-\left(\frac{\pi}{h}+4\right)\right\} \exp \left\{24 \exp \left\{-\left(\frac{\pi}{4}\right)\right\}\right\} .
$$

For $h$ small, $m$ is very small indeed, and all the above series converge extremely rapidly as noted by Fenton and Gardiner-Garden [5]. 
The interpretation of the result (5.1) may be simplified by using expressions $(4.4 a, b)$ and Legendre's identity to show

$$
I=\frac{V^{2}}{\pi}\left(\frac{\pi}{\ln q}+h\right)^{-1}
$$

where $q$ is the nome defined above. For small $h$, this expression may be approximated by

$$
I=\frac{V^{2}}{4 h^{2}}\left(1+\frac{4 h}{\pi}+8 \exp \left\{-\left(\frac{\pi}{h}+4\right)\right\}+\cdots\right)
$$

and this result only differs from the corresponding expression (3.3) by exponentially small terms. For practical purposes, therefore, the simplified model more readily gives the same gap results as the finite plate length problem. Whilst this might have been expected, it is nevertheless pleasing and would appear to be an important conclusion if subsequent work is planned for this problem.

\section{Discussion}

In this paper, the integral $I$ of importance in certain gaseous conductor experiments has been evaluated for a number of cases. For the simplified geometry investigated in Sections 2 and 3, the integral was evaluated with three different sorts of boundary conditions applied to the potential problem. The integral was also evaluated in Sections 4 and 5 when the unsimplified geometry shown in Figure 2(a) was considered.

The main conclusions of the work are twofold. First, under the same boundary conditions, the unsimplified and simplified geometries produce essentially the same value for the integral in the small gap approximation. Secondly, the end effects upon the integral have been obtained, and they are linear with the gap width. Moreover, this linear correction term can be reduced by a factor of three merely by applying different boundary conditions to the upper plate in Figure 1(a) (provided that this is possible experimentally).

The important problem of corner singularities has been neglected in this work apart from their formal appearance in the equations for the transformations. In practice, gaseous conductor experiments are conducted at high voltages and strong electric fields-in the experiments which motivated this work, the upper plate was at $10 \mathrm{kV}$ with respect to the lower plate and the plate separation was about $3 \mathrm{~mm}$. Pitting of the corners of electrodes would be likely, therefore, due to the increase in the electric field towards the corners of the electrodes. This may be overcome using curved electrodes, and the best known of these curved profiles appears to be the Rogowski profile developed from ideas of Maxwell and 
described in reference [3, Section 7.12]. The ideas developed in the present paper suggest another possible use of curved profiles-the minimization of end effects in the value of the integral 1 . Carefully designed perturbations away from the rectangular plates considered here could prove useful, and it is hoped to follow up the ramifications of this suggestion.

\section{Acknowledgement}

The author would like to thank Drs. J. A. Cross and D. Laver for drawing this problem to his attention and Dr. A. N. Stokes for penetrating comments on a first draft.

\section{References}

[1] M. Abramowitz and I. A. Stegun (eds.), Handbook of mathematical functions (Dover, New York, 1965).

[2] G. F. Carrier, M. Krook and C. E. Pearson, Functions of a complex variable (McGraw-Hill, New York, 1966).

[3] J. D. Cobine, Gaseous conductors (Dover, New York, 1941).

[4] N. Davy, "The field between equal semi-infinite rectangular electrodes or magnetic pole pieces", Philos. Mag. (7) 35 (1944), 819-340.

[5] J. D. Fenton and R. S. Gardiner-Garden, "Rapidly convergent method for evaluating elliptic integrals and theta and elliptic functions", J. Austral. Math. Soc. Ser. B 24 (1982), 47-58.

[6] I. S. Gradshteyn and I. M. Ryzhik, Table of integrals, series and products (Academic, New York, 1965).

[7] H. Kober, Dictionary of conformal representations (Dover, New York, 1957).

[8] Lord Rayleigh, "On the theory of resonance", Philos. Trans. Roy. Soc. London 161 (1871), 77-118.

[9] G. F. B. Riemann and H. Weber, Differentialgleichungen der Physik (Brunswick, 1927). 